\title{
Hepatocellular carcinoma: a review of diagnostic challenges for the pathologist
}

This article was published in the following Dove Press journal: Journal of Hepatocellular Carcinoma

\author{
Alberto Quaglia \\ Department of Cellular Pathology, \\ Royal Free Hospital, London NW3 \\ 2QG, UK
}

Correspondence: Alberto Quaglia

Department of Cellular Pathology, Royal Free Hospital, Pond Street, London NW3 2QG, UK

Tel +44 207794 0500ext3564I

Email alberto.quaglia@nhs.net

\begin{abstract}
Histopathologists retain a critical role in the diagnosis and management of hepatocellular carcinoma (HCC). HCC arises usually but not exclusively in a background of advanced-stage chronic liver disease. The histological diagnosis of HCC poses many challenges particularly when dealing with liver biopsy specimens due to the heterogeneity of $\mathrm{HCC}$ and the difficulty to confirm hepatocellular differentiation in some instances. Primary liver tumors should be considered as a continuum with typical hepatocellular and cholangiocarcinoma at the two ends and a whole range of tumors showing both hepatocellular and cholangiocellular differentiation with or without an associated progenitor/stem cell component in the middle. Characterization of combined (or mixed) hepatocellular-cholangiocarcinoma can be very challenging. In advanced-stage chronic liver disease, the main challenge for the histopathologist is still to differentiate between HCC and its precursors, although this is rarely critical in the clinical setting at present. HCC originating in non-cirrhotic livers needs to be differentiated from other primary and extrahepatic tumors and from hepatocellular adenoma, bearing in mind that progression to malignancy is more through a continuum that watertight histological categories.
\end{abstract}

Keywords: hepatocellular carcinoma, hepatocellular adenoma, dysplastic nodule, large regenerative nodule, combined hepatocholangiocarcinoma, mixed hepatocholangiocarcinoma

\section{Introduction}

A disease of multiple facets, hepatocellular carcinoma (HCC), is a major challenge for all involved in its diagnosis and management. Despite considerable recent advances in molecular and imaging techniques, histopathology retains a critical role. The aim of this review is to summarize the main clinical diagnostic scenarios in which HCC poses a diagnostic challenge for the histopathologists (Table 1).

\section{General points}

Advanced stage chronic liver disease (cirrhosis) ${ }^{1}$ is the main risk factor for HCC. Aside combined (or mixed) hepatocellular-cholangiocarcinoma (c-HCC-CCA), pure cholangiocarcinoma can originate in cirrhotic livers and outside chronic biliary disorders but not as frequently as HCC. Metastatic deposits from extrahepatic primaries and primary hepatic mesenchymal tumors (eg, angiomyolipoma) can affect cirrhotic livers but they are rare. When a tumor is found in a cirrhotic liver, it is therefore more likely to be hepatocellular. Depending on its size, the question is usually whether the lesion is a large regenerative nodule (LRN), a dysplastic nodule (DN) with the potential to progress to $\mathrm{HCC}$, or an already transformed HCC. Both focal nodular hyperplasia ${ }^{2}$ and hepato- 
Table I Main HCC diagnostic histological challenges

\begin{tabular}{|l|}
\hline General \\
Tumor heterogeneity \\
Hepatocellular differentiation \\
Mixed (combined) hepatocholangiocarcinoma \\
\hline HCC in advanced-stage chronic liver disease (cirrhosis) \\
Early HCC vs precursors \\
Prognostic factors \\
\hline HCC in normal/near normal liver or early-stage chronic liver \\
disease \\
Differential with non-hepatocellular primary and secondary liver \\
tumors \\
HCC vs hepatocellular adenoma \\
\hline
\end{tabular}

Abbreviation: HCC, hepatocellular carcinoma.

cellular adenoma, ${ }^{3}$ mimicking $\mathrm{HCC}$, have been described in cirrhosis, but they are less common than LRN, DN, and HCC. LRNs, DNs, and HCC in cirrhotic livers are usually diagnosed by imaging and are not biopsied. The pathologist tends to encounter them during the examination of livers removed at transplantation. HCC can also originate in normal or near normal liver or livers with early-stage chronic liver disease, ${ }^{4}$ at all ages. A proportion of these HCC may represent malignant transformation of hepatocellular adenomas. The differential diagnosis in this context is usually between HCC and benign hepatocellular tumors such as hepatocellular adenoma and focal nodular hyperplasia, other primary liver tumors, and in particular, intrahepatic cholangiocarcinoma, and metastatic deposits from extrahepatic primaries. In contrast to $\mathrm{HCC}$ in cirrhosis, these rare HCCs are often biopsied.

\section{Hepatocellular differentiation and tumor heterogeneity}

Regardless of its exact cell of origin (eg, transformed hepatocytes or progenitor/stem cells), the histological diagnosis of HCC is based on the resemblance of tumor cells to hepatocytes. As with any other tumor, the diagnosis is straightforward when HCC is well differentiated, its neoplastic hepatocytes produce bile and are virtually identical to normal ones, showing also the same immunohistochemical profile. It is challenging when lesions are less differentiated, and the neoplastic cells loose their hepatocyte trait or develop changes seen in other tumors (eg, clear cell change).

In addition, HCCs are characteristically heterogeneous. Different areas of the same tumor can show quite different growth patterns and levels of differentiation. Often responsible for a variegated appearance at macroscopic examination, intralesional heterogeneity poses a particular challenge when dealing with liver biopsy specimens, as the changes in a particular sample depend entirely on which area has been harvested by the needle.

In contrast, other primary and extrahepatic tumors can appear "hepatoid" at the microscope. A loose and often abused term, "hepatoid" is used to indicate that tumor cells resemble hepatocytes, based on the way they are arranged, adhere together, and on their nuclear and cytoplasmic appearance. Angiomyolipoma, intrahepatic cholangiocarcinoma, and gallbladder carcinoma spreading into liver, metastatic neuroendocrine tumors, adrenocortical neoplasms, malignant melanoma, metastatic epithelioid gastrointestinal stromal tumors (GIST), chromophobe renal cell carcinoma, clear cell renal carcinoma, and hepatoid adenocarcinoma of gastric or pancreatic origin can look hepatoid and need always to be considered in the differential diagnosis (Figure 1).

The three common HCC growth patterns, trabecular, pseudoglandular (or pseudoacinar), and solid (or compact), can coexist in the same lesion, with sudden and sharp transition from one to the other. Tumor cells can show a variety of changes including fat accumulation, diffuse clarification of their cytoplasm (clear cell change), ground glass inclusions, Mallory-Denk bodies, and oncocytosis. The intervening stroma may be more or less prominent and more or less infiltrated by a variety of leukocytes. In some instances, the prominence and distribution of some of these changes have led to the description of variants or subtypes such as the macrotrabecular massive, ${ }^{5}$ scirrhous (abundant stroma), ${ }^{6}$ clear cell, steatohepatitic (steatosis, ballooning often with prominent Mallory-Denk bodies and pericellular fibrosis), ${ }^{7}$ sarcomatoid, and lymphoepithelioma like. ${ }^{8}$ The coexistence of areas of HCC and cholangiocarcinoma is discussed later.

Bile production is a good indication of hepatocyte differentiation. It is usually in the form of bile plugs of variable size and often in the lumen of acinar or pseudoglandular structures, ranging in color from pale yellow to green or dark brown. When abundant, it is responsible for the green, at times strong, tinge of the tumor cut surface at macroscopic examination. Tumor bile production can be focal, may not be represented in a liver biopsy sample, and is usually hard to find in poorly differentiated areas. It also needs to be differentiated from the bile produced by non-neoplastic hepatocytes entrapped at the tumor periphery, or from mucus, when very light in color.

There are several immunohistochemical markers of hepatocellular differentiation, but they are not entirely specific or sensitive. They can be expressed by other cell lineages and tumors and tend to be lost in poorly differentiated HCCs. ${ }^{9,10}$ HepPar-1 corresponds to the carbamoyl phosphate synthetase 

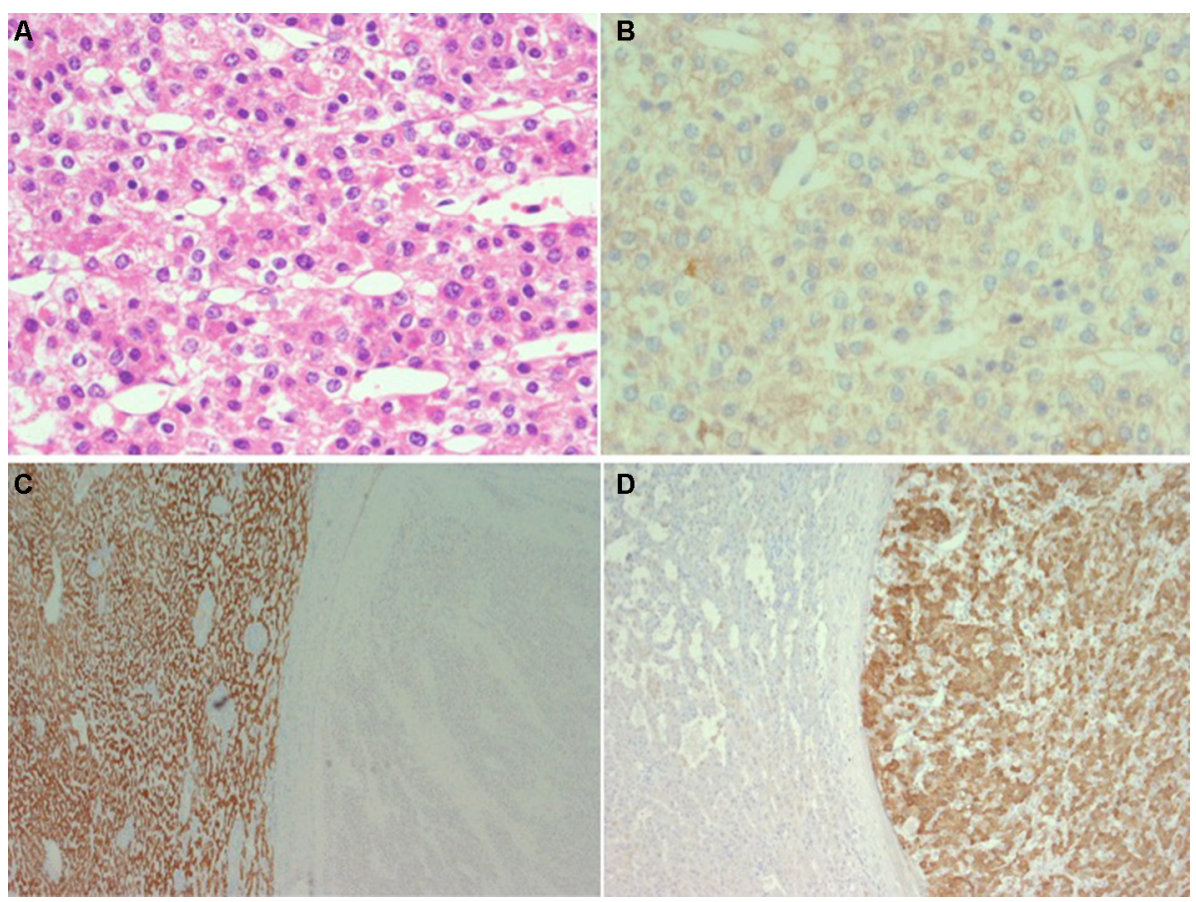

Figure I Eighty-year-old woman with a large intrahepatic tumor in the right lobe suspect for hepatocellular carcinoma.

Notes: Histological examination of the resected specimen shows (A; H\&E) that the tumor is composed of solid sheets or trabeculae of medium to large size cohesive cells with abundant eosinophilic cytoplasm resembling hepatocytes (hepatoid). Immunohistochemistry shows that the tumor cells do not form canaliculi (B; CEA, polyclonal antibody) and do not stain for Hep-Par I (C; background liver on the left and tumor on the right side of the figure) but stain for vimentin (D; same field as C) melan-A, inhibin, synaptophysin and calretinin. The final diagnosis was of liver infiltration by adrenal cortical carcinoma.

1, a urea cycle enzyme located on mitochondria and producing a cytoplasmic staining pattern. Arginase 1 converts arginine to ornithine and urea. Arginase 1 staining is usually cytoplasmic and often nuclear. Mimicking normal hepatocyte function, neoplastic hepatocytes can form canaliculi, which include in their membrane a complex array of transporter proteins, involved in the secretion of the various bile constituents and in the maintenance of the canalicular membrane integrity. Of these proteins, perhaps bile salt export pump (BSEP) is the most specifically expressed by hepatocytes. Staining for BSEP, therefore, has the double advantage of demonstrating canaliculi in addition to a liver-specific antigen. BSEP, however, can produce, at times, a nonspecific cytoplasmic staining pattern (in extrahepatic tumors included, personal observation) with focal linear reinforcement at the membrane that can simulate the formation of canaliculi and has been described to be not entirely specific for HCC. ${ }^{10}$ Other antibodies against canalicular membrane proteins (eg, CD10, polyclonal CEA, and MDR3) can be expressed by other cell lineages, limiting their diagnostic use when staining tumor cells in a more linear or circumferential membranous pattern without clear canalicular polarization. Poorly differentiated, rather than well-differentiated HCC tends to express glypican 3 , an oncofetal protein expressed normally by fetal liver and other tumor lineages. Immunohistochemistry for alpha-feto protein is of limited use due to its heavy background stain, patchy expression, and low specificity and sensitivity. In situ hybridization for albumin is not entirely hepatocyte specific as it has been shown to mark cholangiocarcinomas ${ }^{11}$ and extrahepatic tumors such as acinar cell carcinomas. ${ }^{12}$

In summary, tumor heterogeneity and the lack of entirely specific and sensitive immunohistochemical markers can make the identification of hepatocellular differentiation challenging particularly in biopsy specimens and when HCC is poorly differentiated. Straightforward on H\&E at times, the histological diagnosis of HCC often needs the aid of immunohistochemistry and, in some instances, can only be achieved by clinicopathological correlation. Other primary and extrahepatic tumors metastasizing to the liver can mimic HCC and need always to be considered in the differential diagnosis, particularly in noncirrhotic patients.

\section{c-HCC-CCA}

The term c-HCC-CCA refers to malignant epithelial liver tumors in which HCC and cholangiocarcinoma coexist (Figure 2). The criteria for the diagnosis of this entity have changed over the years and have included the occurrence of HCC and cholangiocellular carcinoma as separate lesions 


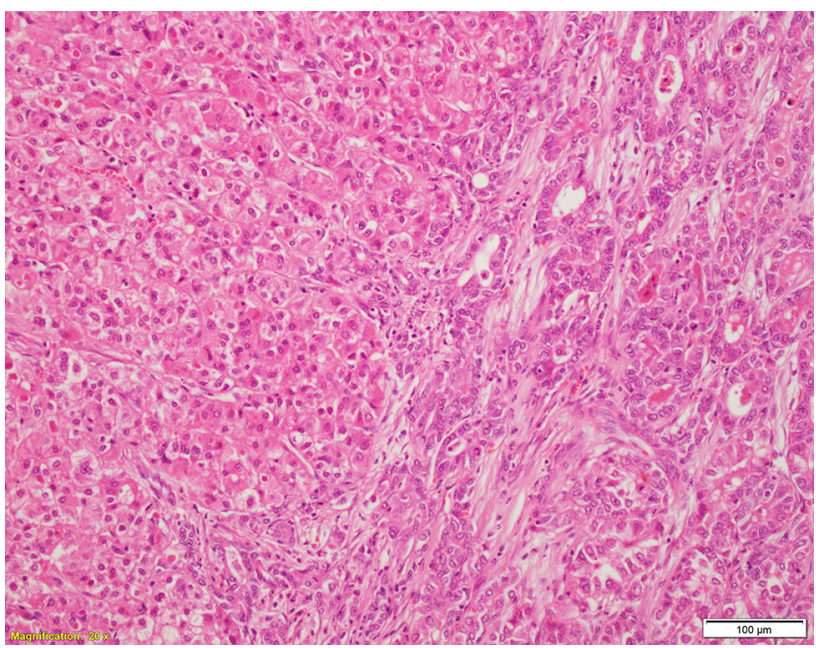

Figure 2 c-HCC-CCA, classic type with the hepatocellular component on the left side and the cholangiocellular component on the right side (H\&E).

Abbreviation: c-HCC-CCA, combined (or mixed) hepatocellular-cholangiocarcinoma.

in the same liver, the collision of separate foci of HCC and cholangiocellular carcinoma, and the presence of hepatocellular and cholangiocellular differentiation in a single lesion. c-HCC-CCA is considered to be a particularly aggressive form of primary liver cancer. Based on notions derived from liver embryology, animal models of liver carcinogenesis and regeneration, studies on the hepatic progenitor/stem cell compartment, and new concepts on cell plasticity, there is now the tendency to view primary liver tumors as part of a spectrum with typical, pure HCC and cholangiocarcinoma at the two ends and a plethora of tumors showing features of both in the middle. ${ }^{13}$ The exact cell of origin behind this tumor spectrum remains to be defined, with candidates including transformed hepatocytes or cholangiocytes acquiring stem cell features, regression to hepatoblasts, and transformed progenitor/stem cell maintaining dual differentiation potential. The observation that c-HCC-CCA is often associated with previous local ablation therapy and in particular trans-arterial chemoembolization ${ }^{14}$ raises the possibility that therapeutic intervention can unmask the complexity of these tumors and give a selective growth advantage to resistant tumor cells.

The WHO 2010 classification of tumors of the digestive system ${ }^{15}$ separates c-HCC-CCA into two groups, classic type and c-HCC-CCA with stem cell features. Coexistence of $\mathrm{HCC}$ and cholangiocarcinoma arising as separate foci, with or without collision, in the same liver, hepatoblastoma, and fibrolamellar carcinoma is not included in the WHO 2010 definition of c-HCC-CCA. In the classic type, areas of typical HCC coexist with areas of typical cholangiocarcinoma. c-HCC-CCA with stem cell features is further sub-classified into three subtypes (typical, intermediate, and cholangiolocellular). The typical subtype consists of nests of hepatoid cells in abundant stroma along with a cuff of oval-like cells, which express progenitor cell markers. The intermediate subtype is composed of a monomorphic population of cells with an intermediate morphology between hepatocytes and cholangiocytes and expressing markers of both. The cholangiolocellular-type is made of tubular anastomosing antler-like structures in abundant fibrous stroma and expressing progenitor cell markers. The histological diagnosis of c-HCC-CCA, and in particular the stem cell feature variant, can be very challenging. This is because immunohistochemical markers of progenitor/stem cells are not entirely sensitive or specific of progenitor stem cells. The morphological appearance and immunohistochemical profile of a putative progenitor/ stem cell component can also overlap considerably with the complex spectrum of phenotypes and levels of differentiation of typical HCC and cholangiocarcinoma.

A recent international consensus ${ }^{16}$ has proposed a new set of terms and criteria to define c-HCC-CCA. This document recognizes the need to reconsider whether the cholangiolocellular and intermediate subtypes of the WHO 2010 classification should be part of c-HCC-CCA or separate entities. Rather than constituting a formal subtype, the presence of stem/cell progenitor cell features should simply be described by the pathologist.

Emphasis in this document is placed on the need to classify c-HCC-CCA on the basis of the appearance on routine sections including $\mathrm{H} \& \mathrm{E}$ and histochemical stains (for matrix proteins and mucins). Immunohistochemistry should be used as a supporting tool to characterize hepatocellular differentiation and cholangiocellular differentiation. This implies that the expression by morphologically typical HCC of markers of cholangiocytic differentiation or progenitor stem cells or the expression by morphologically typical cholangiocarcinoma of markers of hepatocellular differentiation or progenitor/stem cells should not be used as the evidence of c-HCC-CCA.

The consensus recognizes the challenge posed by the interpretation of progenitor/stem cell markers, with particular reference to the similarities between the cholangiocellular lineage and the progenitor/stem cell compartment, due to the close anatomical and functional relationships between the biliary tree and the stem cell/progenitor niche. When a tumor expresses markers shared by cholangiocytes and stem/progenitor cells, the diagnosis should be based on the morphological appearance (ie, cholangiocarcinoma if the cells are forming tubuloglandular structures). In contrast, staining for stem cell/progenitor markers, not expressed by 
mature cholangiocytes, should be considered sufficient for the identification of a stem/progenitor cell component.

Of critical importance for patient management is the refinement of the criteria to identify or at least to suspect c-HCC-CCA by imaging. Current data suggest that c-HCCCCA has a radiological appearance overlapping with that of intrahepatic cholangiocarcinoma. A discrepancy between the radiological appearance and tumor markers alpha-fetoprotein (AFP) and carbohydrate antigen 19-9 (Ca 19-9) or a simultaneous increase in both AFP and CA 19-9 should raise the suspicion of a c-HCC-CCA.

The histological diagnosis of c-HCC-CCA is particularly challenging on liver biopsy because of potential sampling error. More studies are necessary to clarify how extensively and in which parts a primary liver tumor should be sampled in order to confirm or exclude c-HCC-CCA. Extensive sampling of surgically resected primary epithelial liver tumors therefore becomes critical to inform on the true incidence and morphological spectrum of c-HCC-CCA and to correlate with clinical, radiological, and molecular features. This is also important from a prognostic viewpoint, given the unpredictable behavior of c-HCC-CCA when spreading to extrahepatic sites. ${ }^{17}$

\section{$\mathrm{HCC}$ in patients with advanced-stage chronic liver disease (cirrhosis)}

Advanced-stage chronic liver disease, cirrhosis, ${ }^{1}$ remains the main risk factor for $\mathrm{HCC}$. The development of $\mathrm{HCC}$ in cirrhotic liver is thought to occur in stages from regenerative nodules through DNs. DNs are small size nodules of about 10-20 mm diameter, which in comparison to regenerative nodules and LRNs show some atypical histological features but fall short of HCC. DNs are considered to be neoplastic and the true precursor stage of HCC for a number of reasons including that 1) they tend to be identified in livers containing one or more separate foci of overt $\mathrm{HCC}$; 2) some nodules resembling DNs contain foci of overt HCC (nodule-in-nodule pattern) suggesting the progression of neoplastic clones; 3) DNs show molecular similarities with $\mathrm{HCC}$; and 4) when biopsied and followed-up on imaging, they tend to transform into HCC. ${ }^{18,19}$

The transition from DN to HCC is thought to be associated with a change in the lesional vascular supply, from a dual portal-arterial to a predominantly arterial, due to neoangiogenesis. The histological counterpart to this change is the presence inside the lesion of unpaired arteries ${ }^{20}$ associated with modifications of the endothelium lining the sinusoids, which looses its fenestration and expresses markers usually expressed by nonsinusoidal endothelium (eg, CD34). The radiological counterpart of these changes is contrast uptake in the arterial phase and rapid wash-out in the venous phase, considered to be sufficient for a diagnosis of $\mathrm{HCC}$, and which has reduced the need of liver biopsy in the last decade. ${ }^{21} \mathrm{~A}$ small proportion of $\mathrm{HCC}$, however, may be hypovascular, and a small proportion of nodules with radiological features suggestive of HCC may not be hepatocellular. ${ }^{22}$ An international consensus in $2006^{23}$ highlighted the difficulties in separating histologically the different stages of HCC progression particularly in differentiating low-grade DNs from LRNs and high-grade DNs from early HCC probably because they simply represent part of a continuum. Stromal invasion defined as the presence of tumor cells inside portal tracts or fibrous septa has been proposed, but not widely accepted, as a diagnostic feature of HCC. More recently, correlation with molecular studies has led to the introduction of markers that can be used immunohistochemically to characterize these lesions in more detail. Heath-shock protein 70, glypican 3, and glutamine synthetase were the first to be proposed in differentiating $\mathrm{DNs}$ from overt $\mathrm{HCC} .{ }^{24}$ Lesions not staining with any of these three markers were unlikely to be highgrade DN. Positive staining for one marker in isolation was considered supportive of well-differentiated HCC without excluding a high-grade $\mathrm{DN}$, and staining for two or three markers were considered in favor of well-differentiated HCC over high-grade DN. The view on $\mathrm{HCC}$ and its precursor lesions has gradually changed from the concept of watertight categories to phases of progression, marked by an overlapping phenotypical change marked by the following three phases: 1) high-grade DN iso- or hypovascularized with individual arteries and portal perfusion and without stromal invasion histologically; 2) early HCC, with residual portal perfusion, and iso-hypovascularity on imaging, histologically well differentiated, vaguely nodular with indistinct margins, containing individual arteries, with or without stromal invasion; and 3) progressed HCC, with characteristic imaging features of hypervascularity in the arterial phase and hypovascularity in the venous phase, histologically moderately differentiated, distinctly nodular, with or without stromal invasion vascular invasion, including individual arteries but no portal tracts. ${ }^{23}$ Along similar lines, more recent studies have shown that the combination of molecular techniques and histological and immunohistochemical features can help in gauging where a nodular hepatocellular lesion is positioned in the regenerative to malignant spectrum of progression..$^{25,26}$

As a result, liver biopsy is fallen out of favor in recent years as a tool for the diagnosis of HCC in advanced-stage chronic liver disease and in particular early small lesions, 
due essentially to: 1) the acceptance of radiological criteria as sufficient for the diagnosis of HCC; 2) limited histological criteria to differentiate between LRNs and low-grade DNs and between high-grade DNs and well-differentiated HCC and consequent poor interobserver reproducibility (in addition, the widespread adoption of local ablation therapy has reduced the impact of these relatively fine distinctions on clinical management; small lesions are now biopsied often in the same session of local ablation, and by the time the specimen reaches the laboratory, the lesion has already been destroyed); 3) lack of validated molecular data; and 4) the clinical perception that morbidity, mortality, and seeding risk outweighed the low diagnostic histological yield. Liver biopsy however may become fashionable again as the accuracy of cross-sectional imaging in the diagnosis of early HCC is now questioned, and there are now additional immunohistochemical and molecular tools available to the histopathologists. ${ }^{25,26}$ Liver biopsy may be particularly helpful in characterizing those hepatocellular lesions, such as focal nodular hyperplasia and hepatocellular adenoma, which rarely occur in the context of cirrhosis and can create issues with the differential diagnosis of HCC particularly when of relatively large size. The use of liver biopsy has been advocated in clinical trials' setting. ${ }^{27}$ The limitations however of liver biopsy remain, as a snapshot of part of a lesion at a particular point in time.

Examination of surgical specimens, and in particular whole livers removed at transplantation, remains a routine diagnostic procedure. The issue related to the differential diagnosis between precursor lesions and HCC is somewhat tempered, as the clinical emphasis in this context is to confirm the preoperative diagnosis of HCC, assess the effects of preoperative local ablation therapies, confirm or re-evaluate the tumor staging at listing in case of interval tumor progression, and identify prognostic factors, which can inform on the potential clinical course after transplantation, and in particular on the risk of tumor recurrence.

It remains important, however, to sample and analyze HCC and its precursors in a systematic way, for our better understanding of hepatocarcinogenesis, and to validate and improve the sensitivity and specificity of imaging techniques. ${ }^{28}$ Small lesions still escape pretransplantation detection. An extreme example is the so-called (rare) cirrhotomimetic variant of $\mathrm{HCC}$, in which multiple minute HCC nodules, of the size of regenerative nodules, are scattered through the parenchyma. If not associated with larger dominant tumors, cirrhotomimetic HCC is well below the threshold for imaging detection and is an exclusive histo- logical diagnosis at present. A proportion of cirrhotomimetic HCC have an aggressive post-transplant course. ${ }^{29}$

Explanted livers should be cut into thin slices, ideally less than $10 \mathrm{~mm}$ in thickness, to minimize the risk of missing small nodular lesions. Tumors should be sampled extensively to include any area with a distinct appearance. The correlation between the size, number, and position of hepatocellular tumor and preoperative imaging and ablation therapy can be challenging. It may be hard to reproduce accurately the computerised tomography (CT) plane when slicing the liver, even on a transverse plan. Properly thin slices are obtained more easily after formalin fixation, which causes distortion and shrinking of the specimen modifying its original shape. Interval tumor progression or partial or complete regression may have taken place between the time of imaging, local ablation, and transplantation.

Vascular invasion has been consistently found to be associated with poor prognosis after transplantation. Its identification, however, is not always straightforward. HCC is well vascularized, and because of the intimate relationship between hepatocytes and their sinusoidal network, it is often difficult to differentiate between invaded and ambient vascular structures. Similar to other tumors (eg, renal cell carcinoma and follicular carcinoma of the thyroid), vascular invasion in HCC is usually in the form of an intravascular intraluminal growth, rather than destructive infiltration, aided by its sinusoidal endothelial coat, which separates tumor cells from blood. ${ }^{30}$ Once a vascular structure is fully occupied and obliterated by tumor, it remodels and may not be recognizable any longer. The tumor nodular deposit can therefore appear as a tumor focus embedded in fibrous stroma, often as one or more "satellite" nodules at the tumor periphery. This creates issues in differentiating between intrahepatic spread and multifocal disease as part of a field change. Changes in favor of local spread include: 1) the location of tumor deposits within a portal tract remnant; 2) monomorphic tumor deposits composed of moderately or poorly differentiated areas (lack of well-differentiated areas, or changes suggestive of a nodule-in-nodule pattern, argues against the deposit to be a separate early HCC); 3 ) a cluster of satellite nodules with the same morphology; and 4) lack of DNs or early HCC in other parts of the same liver. ${ }^{31}$ The use of additional histochemical and immunohistochemical stains, along with extensive sampling at the tumor periphery, has been proposed to minimize the risk of missing vascular invasion and identify vascular remnants. ${ }^{32}$ This approach, however, may not be feasible in clinical diagnostic practice, as it may have a considerable impact on the laboratory diagnostic workload and turnaround time. 
The risk of HCC in patients with advanced-stage chronic liver disease depends on a number of factors, including gender and the underlying disease etiology. It is not possible to date to predict histologically the risk of developing $\mathrm{HCC}$ based on changes present in the background liver. Small cell change and large cell change, iron-free foci in livers of patients with genetic hemochromatosis, ${ }^{33}$ areas of severe irregular regeneration of hepatocytes ${ }^{34}$ could represent the early prelesional signs of potential HCC development, but it remains unclear whether a specific risk can be quantified, or whether and how they could dictate patient stratification or clinical management.

\section{Patients with normal or near normal liver, or early-stage chronic liver disease and $\mathrm{HCC}$}

HCC can arise in livers not affected by advanced-stage chronic liver disease (noncirrhotic livers) at any age. A somewhat loose definition, the term "non-cirrhotic" includes normal livers, livers with mild changes, and livers affected by substantial fibrosis in the context of various types of chronic liver injury. ${ }^{4}$ An open question in some patients with underlying chronic liver disease and severe fibrosis is whether HCC anticipated progression to cirrhosis, or whether fibrosis improved in response to therapy (eg, viral hepatitis, hemochromatosis) without eliminating the risk of neoplastic progression. These tumors may remain asymptomatic for a long time and reach a large size, because patients do not present earlier with symptoms of advanced-stage chronic liver disease. Common associated factors include hepatitis $\mathrm{B}$ virus infection, nonalcoholic steatohepatitis, genetic hemochromatosis, other metabolic disorders including glycogen storage disease, alpha-1-antitrypsin deficiency, and porphyria, sex hormones, and in particular, anabolic steroids, vascular disorders such as Budd-Chiari and portosystemic shunts, and exposure to toxins (eg, aflatoxin B1). In a recent study, ${ }^{35}$ surgically resected HCC could be separated into two main categories based on the correlation of histological and molecular features. One type consisted of large well-differentiated cholestatic tumors in the pseudoglandular and microtrabecular patterns, showing mutations in CTNNB1, the gene encoding for beta-catenin. The other type consisted of poorly differentiated tumors, displaying a compact growth pattern, with frequent vascular invasion, and showing mutation in TP53. Other specific mutations were identified in the scirrhous and steatohepatitic variants. A novel subtype defined as "macrotrabecular massive" due to the particular growth pattern showed vascular invasion, high serum AFP, mutation in TP53 and FGF19 amplifications, and poor survival.

The questions posed to the histopathologists in this context are usually 1) to confirm that the tumor is HCC rather than cholangiocarcinoma or a metastasis from an extrahepatic primary and 2) to clarify whether a suspected hepatocellular lesion is benign (hepatocellular adenoma) or malignant (HCC).

\section{Is the tumor hepatocellular?}

The identification of a large mass in a patient without a clinical history of liver disease usually raises the question of its nature, particularly in an elderly patient and if serum AFP is not elevated. A biopsy is usually required to confirm the diagnosis. As mentioned earlier, there are a number of primary hepatic tumors and metastatic tumor deposits that can mimic HCC histologically. Intrahepatic (peripheral) cholangiocarcinoma, in particular, is typically intraparenchymal and mass forming. Histologically, it is often characterized by areas composed of cords or sheets of large cells with eosinophilic cytoplasm and an overall hepatoid appearance. Lack of bile production, presence of mucin, lack of canaliculi by immunohistochemistry, cytoplasmic staining for CEA, CA 19-9, and biliary cytokeratin, and lack of expression of Hep-Par 1 or arginase 1 are in favor of cholangiocarcinoma. Gallbladder carcinoma infiltrating into liver parenchyma can appear hepatoid and can enter the differential diagnosis when the tumor front is biopsied. As mentioned earlier, angiomyolipoma, metastatic neuroendocrine tumor, adrenocortical tumor, malignant melanoma, metastatic epithelioid GIST, chromophobe renal cell carcinoma, clear cell renal carcinoma, and hepatoid adenocarcinoma of gastric or pancreatic origin can simulate HCC and need always to be considered in the differential diagnosis. A detailed description of the immunohistochemical profiling necessary to distinguish between HCC and any of these tumors is outside the scope of this review.

The differential diagnosis of large AFP protein secreting tumors arising in the liver of patients without the history or signs of chronic liver disease or a metabolic disorder includes HCC, hepatoblastoma, the so-called transitional liver tumor (see below), germ cell tumors, and AFP-secreting carcinoma of gastrointestinal origin or pancreatic tumors such as acinar cell carcinoma and pancreatoblastoma. This differential is particularly challenging when liver biopsy samples include just poorly differentiated areas. Hepatic germ cell tumors are very rare. Similarly to extragonadal retroperitoneal tumors, ${ }^{36}$ they could represent metastasis from occult or regressed, primary gonadal tumors. Teratomas $\mathrm{s}^{37-40}$ and yolk sac tumors ${ }^{41-43}$ 
are the most common types of hepatic germ cell tumors and can occur at any age. Instances of mixed tumors including teratomatous, yolk sac, choriocarcinoma, and embryonal carcinoma elements have been described in both adults and children. ${ }^{44-46}$ Of note, hepatocellular differentiation can be observed in germ cell tumors. ${ }^{47}$

Fibrolamellar carcinoma can pose a diagnostic challenge, particularly on liver biopsy specimens in which not all typical features may be represented. Fibrolamellar carcinoma can include areas resembling more conventional HCC. Conventional HCCs can simulate fibrolamellar carcinoma, when stroma is abundant. The recent identification of the DNAJB1-PRKACA fusion can help in the differential diagnosis and can be performed by fluorescence in situ hybridization (FISH), ${ }^{48}$ which is however not widely available and may not be cost-effective to develop outside referral centers given that this tumor is rare.

\section{Is this hepatocellular tumor benign or malignant, and in particular, is this a hepatocellular adenoma or a $\mathrm{HCC}$ ?}

This question relates essentially to the similarities between the beta-catenin-activated type of hepatocellular adenoma and well-differentiated HCC. Beta-catenin-activated hepa- tocellular adenoma ${ }^{49}$ affects often men and shows atypical histological features such as small cell change, nuclear atypia, and pseudoglandular change that can also be observed in well-differentiated HCC. Evidence of beta-catenin activation is usually in the form of aberrant nuclear staining of beta-catenin (rather than the membranous pattern observed in normal hepatocytes or adenomas of other types), but this change can be very focal and not present in a liver biopsy sample. Diffuse strong staining for glutamine synthetase (the enzyme involved in ammonia detoxification, a downstream beta-catenin target, and normally expressed by perivenular hepatocytes) is generally used as a surrogate marker of betacatenin activation. In some instances, however, the glutamine synthetase staining is patchy and may not correlate well with mutations in CTNNB1. Beta-catenin-activated HCA can coexist with HCC as separate foci in the same liver, and hepatocellular adenoma-like areas may be present at the periphery of otherwise overt HCC. Beta-catenin-activated hepatocellular adenoma and well-differentiated HCC are probably part of a continuum, in which multiple mutational hits are responsible for neoplastic progression, ${ }^{50}$ rather than separate entities. This may also apply to other adenoma subtypes. About $10 \%$ of inflammatory hepatocellular adenoma, for example, harbor mutation in the CTNNB1 and are also

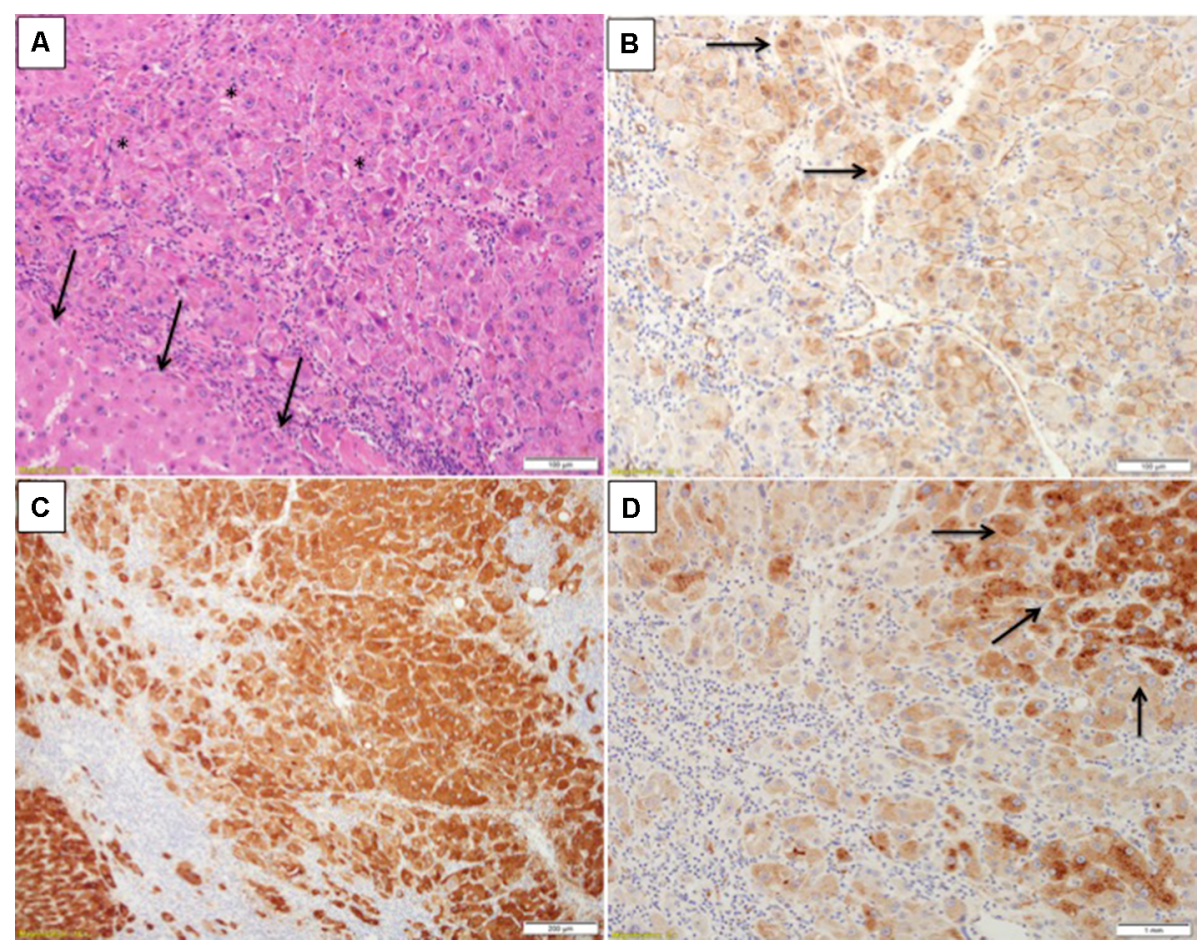

Figure 3 Inflammatory hepatocellular adenoma with focal signs of concomitant beta-catenin activation.

Notes: (A) H\&E section showed an area of increased cell density (asterisks) in comparison to a nearby one in which hepatocytes are more of normal size (arrows). (B) Beta-catenin immunostain from the same area shows nuclear staining in places (arrows). (C) Glutamine synthetase is diffusely expressed in this area. (D) Serum amyloid $A$ is expressed by nearby hepatocytes (arrows). Courtesy Dr Andrew Drake, Christchurch, New Zealand. 
at risk of malignant transformation (Figure 3). The term "hepatocellular neoplasm with uncertain malignant potential (HUMP)" has been proposed ${ }^{51,52}$ for those borderline atypical lesions in which a definitive histological diagnosis of HCC cannot be made, particularly with a liver biopsy. The context in which hepatocellular adenoma occurs is critical. An indolent looking steatotic hepatocellular adenoma with features of an HNF-1-mutated variant in a young healthy woman taking oral contraceptives and with an entirely normal background liver is probably at very low risk of progression and may regress once hormonal stimulation is withdrawn. In contrast, a hepatocellular lesion with the appearance of a hepatocellular adenoma on liver biopsy, with or without atypical features, arising in a postmenopausal woman, in a man, in an individual taking anabolic steroids, or in patients with underlying vascular disorder, steatohepatitis, or other disorders such as glycogen storage disease, should be considered at risk of neoplastic progression.

Contributing to the challenge of predicting histologically the behavior of hepatocellular tumors is the instance of histologically overt $\mathrm{HCC}$, regressing or even disappearing after the withdrawal of hormonal stimulation, as in the cases described by McCaughan et al. ${ }^{53}$

\section{HCC and hepatoblastoma}

The distinction between HCC and hepatoblastoma can be difficult. Hepatoblastoma tends to affect children in the first 2 years of their life. HCC usually affects older children, a proportion of whom have an underlying inherited metabolic disorder. The macrotrabecular variant of hepatoblastoma can resemble HCC very closely. Transitional liver cell tumor ${ }^{54}$ is the term used to describe tumors with features overlapping between hepatoblastoma and HCC, affecting children older than 2 years or adolescents. Whether transitional liver cell tumor represents a specific subtype or is simply a variant of HCC remains, in my opinion, unclear.

\section{Conclusion}

The histopathologist still plays a critical role in the diagnosis of HCC, in various clinical scenarios, despite recent advances in molecular biology and imaging techniques. The diagnostic challenges for the histopathologist relate essentially to the biological complexity of HCC, including its heterogeneity, the difficulty at times to define hepatocellular differentiation histologically, and the presence of a continuum rather than watertight categories when considering progression to malignancy and the cellular plasticity of primary liver tumors in general.

\section{Disclosure}

The author reports no conflicts of interest in this work.

\section{References}

1. Hytiroglou P, Snover DC, Alves V, et al. Beyond "cirrhosis": a proposal from the International Liver Pathology Study Group. Am J Clin Pathol. 2012;137(1):5-9.

2. Quaglia A, Tibballs J, Grasso A, et al. Focal nodular hyperplasia-like areas in cirrhosis. Histopathology. 2003;42(1):14-21.

3. Calderaro J, Nault JC, Balabaud C, et al. Inflammatory hepatocellular adenomas developed in the setting of chronic liver disease and cirrhosis. Mod Pathol. 2016;29(1):43-50.

4. Blanc JF, De Ledinghen V, Bernard PH, et al. Increased incidence of HFE C282Y mutations in patients with iron overload and hepatocellular carcinoma developed in non-cirrhotic liver. J Hepatol. 2000;32(5):805-811.

5. Ziol M, Poté N, Amaddeo G, et al. Macrotrabecular-massive hepatocellular carcinoma: A distinctive histological subtype with clinical relevance. Hepatology. 2018;68(1):103-112.

6. Okamura N, Yoshida M, Shibuya A, Sugiura H, Okayasu I, Ohbu M. Cellular and stromal characteristics in the scirrhous hepatocellular carcinoma: comparison with hepatocellular carcinomas and intrahepatic cholangiocarcinomas. Pathol Int. 2005;55(11):724-731.

7. Salomao M, Yu WM, Brown RS Jr, Emond JC, Lefkowitch JH. Steatohepatitic hepatocellular carcinoma (SH-HCC): a distinctive histological variant of $\mathrm{HCC}$ in hepatitis $\mathrm{C}$ virus-related cirrhosis with associated NAFLD/NASH. Am J Surg Pathol. 2010;34(11):1630-1636.

8. Si MW, Thorson JA, Lauwers GY, DalCin P, Furman J. Hepatocellular lymphoepithelioma-like carcinoma associated with epstein barr virus: a hitherto unrecognized entity. Diagn Mol Pathol. 2004;13(3):183-189.

9. Lagana SM, Salomao M, Remotti HE, Knisely AS, Moreira RK. Bile salt export pump: a sensitive and specific immunohistochemical marker of hepatocellular carcinoma. Histopathology. 2015;66(4):598-602.

10. Fujikura K, Yamasaki T, Otani K, et al. BSEP and MDR3: Useful Immunohistochemical Markers to Discriminate Hepatocellular Carcinomas From Intrahepatic Cholangiocarcinomas and Hepatoid Carcinomas. Am J Surg Pathol. 2016;40(5):689-696.

11. Shahid M, Mubeen A, Tse J, et al. Branched chain in situ hybridization for albumin as a marker of hepatocellular differentiation: evaluation of manual and automated in situ hybridization platforms. Am J Surg Pathol. 2015;39(1):25-34.

12. Terris B, Hergli I, Lin-Marq N, Rubbia-Brandt L. Letter to the editor with regard to the article entitled: "branched chain in situ hybridization for albumin as a marker of hepatocellular differentiation". Am J Surg Pathol. 2015;39(8):1156-1157.

13. Brunt EM, Paradis V, Sempoux C, Theise ND. Biphenotypic (hepatobiliary) primary liver carcinomas: the work in progress. Hepat Oncol. 2015;2:255-273.

14. Zen C, Zen Y, Mitry RR, et al. Mixed phenotype hepatocellular carcinoma after transarterial chemoembolization and liver transplantation. Liver Transpl. 2011;17(8):943-954.

15. Theise ND, Park YN. Nakanuma Y. Combined hepatocellularcholangiocarcinoma. In: Bosman FT, Carneiro F, Hruban RH, Theise ND, editors. WHO Classification of Digestive Tumors. 4th ed. IARC; 2010.

16. Brunt E, Aishima S, Clavien PA, et al. cHCC-CCA: Consensus terminology for primary liver carcinomas with both hepatocytic and cholangiocytic differentation. Hepatology. 2018;68(1):113-126.

17. De Vito C, Sarker D, Ross P, Heaton N, Quaglia A. Histological heterogeneity in primary and metastatic classic combined hepatocellular-cholangiocarcinoma: a case series. Virchows Arch. 2017;471(5): 619-629.

18. Terasaki S, Kaneko S, Kobayashi K, Nonomura A, Nakanuma Y. Histological features predicting malignant transformation of nonmalignant hepatocellular nodules: a prospective study. Gastroenterology. 1998;115(5):1216-1222. 
19. Borzio M, Fargion S, Borzio F, et al. Impact of large regenerative, low grade and high grade dysplastic nodules in hepatocellular carcinoma development. J Hepatol. 2003;39(2):208-214.

20. Himeno H, Enzan H, Saibara T, Onishi S, Yamamoto Y. Hitherto unrecognized arterioles within hepatocellular carcinoma. J Pathol. 1994;174(3):217-222.

21. Llovet JM, Bruix J. Novel advancements in the management of hepatocellular carcinoma in 2008. J Hepatol. 2008;48(Suppl 1):S20-S37.

22. Caturelli E, Solmi L, Anti M, et al. Ultrasound guided fine needle biopsy of early hepatocellular carcinoma complicating liver cirrhosis: a multicentre study. Gut. 2004;53(9):1356-1362.

23. International Consensus Group for Hepatocellular Neoplasia. Pathologic diagnosis of early hepatocellular carcinoma: a report of the international consensus group for hepatocellular neoplasia. Hepatology. 2009;49(2):658-664.

24. Di Tommaso L, Franchi G, Park YN, et al. Diagnostic value of HSP70, glypican 3, and glutamine synthetase in hepatocellular nodules in cirrhosis. Hepatology. 2007;45(3):725-734.

25. Sciarra A, Di Tommaso L, Nakano M, et al. Morphophenotypic changes in human multistep hepatocarcinogenesis with translational implications. J Hepatol. 2016;64(1):87-93.

26. Nault JC, Calderaro J, Di Tommaso L, et al. Telomerase reverse transcriptase promoter mutation is an early somatic genetic alteration in the transformation of premalignant nodules in hepatocellular carcinoma on cirrhosis. Hepatology. 2014;60(6):1983-1992.

27. Torbenson M, Schirmacher P. Liver cancer biopsy - back to the future?! Hepatology. 2015;61(2):431-433.

28. Müllhaupt B, Durand F, Roskams T, Dutkowski P, Heim M. Is tumor biopsy necessary? Liver Transpl. 2011;17(Suppl 2):S14-S25.

29. Clayton EF, Malik S, Bonnel A, et al. Liver transplantation and cirrhotomimetic hepatocellular carcinoma: classification and outcomes. Liver Transpl. 2014;20(7):765-774.

30. Quaglia A, Etessami N, Sim R, Difford J, Dhillon AP. Vascular invasion and herniation by hepatocellular carcinoma in cirrhosis: A wolf in sheep's clothing? Arch Pathol Lab Med. 2005;129(5):639-644.

31. Kojiro M, Roskams T. Early hepatocellular carcinoma and dysplastic nodules. Semin Liver Dis. 2005;25(2):133-142.

32. Roayaie S, Blume IN, Thung SN, et al. A system of classifying microvascular invasion to predict outcome after resection in patients with hepatocellular carcinoma. Gastroenterology. 2009;137(3):850-855.

33. Deugnier YM, Charalambous P, Le Quilleuc D, et al. Preneoplastic significance of hepatic iron-free foci in genetic hemochromatosis: a study of 185 patients. Hepatology. 1993;18(6):1363-1369.

34. Shibata M, Morizane T, Uchida T, et al. Irregular regeneration of hepatocytes and risk of hepatocellular carcinoma in chronic hepatitis and cirrhosis with hepatitis-C-virus infection. Lancet. 1998;351(9118): 1773-1777.

35. Calderaro J, Couchy G, Imbeaud S, et al. Histological subtypes of hepatocellular carcinoma are related to gene mutations and molecular tumour classification. J Hepatol. 2017;67(4):727-738.
36. Williamson SR, Delahunt B, Magi-Galluzzi C, et al. The World Health Organization 2016 classification of testicular germ cell tumours: a review and update from the International Society of Urological Pathology Testis Consultation Panel. Histopathology. 2017;70(3):335-346.

37. Witte DP, Kissane JM, Askin FB. Hepatic teratomas in children. Pediatr Pathol. 1983;1(1):81-92.

38. Winter TC 3rd, Freeny P. Hepatic teratoma in an adult. Case report with a review of the literature. J Clin Gastroenterol. 1993;17(4):308-310.

39. Watanabe I, Kasai M, Suzuki S. True teratoma of the liver--report of a case and review of the literature--. Acta Hepatogastroenterol (Stuttg). 1978;25(1):40-44.

40. Todani T, Tabuchi K, WatanabeY, Tsutsumi A. True hepatic teratoma with high alpha fetoprotein in serum. J Pediatr Surg. 1977;12(4):591-592.

41. Wong NA, D'Costa H, Barry RE, Alderson D, Moorghen M. Primary yolk sac tumour of the liver in adulthood. J Clin Pathol. 1998;51(12):939-940.

42. Warren M, Thompson KS. Two cases of primary yolk sac tumor of the liver in childhood: case reports and literature review. Pediatr Dev Pathol. 2009;12(5):410-416.

43. Wakely PE Jr, Krummel TM, Johnson DE. Yolk sac tumor of the liver. Mod Pathol. 1991;4(1):121-125.

44. Xu AM, Gong SJ, Song WH, et al. Primary mixed germ cell tumor of the liver with sarcomatous components. World $J$ Gastroenterol. 2010;16(5):652-656.

45. Verma M, Agarwal S, Mohta A. Primary mixed germ cell tumour of the liver--a case report. Indian J Pathol Microbiol. 2003;46(4):658-659.

46. Theegarten D, Reinacher A, Graeven U, Philippou S. Mixed malignant germ cell tumour of the liver. Virchows Arch. 1998;433(1):93-96.

47. Nakashima N, Fukatsu T, Nagasaka T, Sobue M, Takeuchi J. The frequency and histology of hepatic tissue in germ cell tumors. Am J Surg Pathol. 1987;11(9):682-683.

48. Graham RP, Jin L, Knutson DL, et al. DNAJB1-PRKACA is specific for fibrolamellar carcinoma. Mod Pathol. 2015;28(6):822-829.

49. Bioulac-Sage P, Sempoux C, Balabaud C. Hepatocellular adenomas: morphology and genomics. Gastroenterol Clin North Am. 2017;46(2):253-272.

50. Rebouissou S, Franconi A, Calderaro J, et al. Genotype-phenotype correlation of CTNNB1 mutations reveals different $\beta$-catenin activity associated with liver tumor progression. Hepatology. 2016;64(6):2047-2061.

51. Bedossa P, Burt AD, Brunt EM, et al. Well-differentiated hepatocellular neoplasm of uncertain malignant potential: proposal for a new diagnostic category. Hum Pathol. 2014;45(3):658-660.

52. Balabaud C, Bioulac-Sage P, Ferrell L, et al. Well-differentiated hepatocellular neoplasm of uncertain malignant potential. Hum Pathol. 2015;46(4):634-635.

53. McCaughan GW, Bilous MJ, Gallagher ND. Long-term survival with tumor regression in androgen-induced liver tumors. Cancer. 1985;56(11):2622-2626.

54. Prokurat A, Kluge P, Kościesza A, Perek D, Kappeler A, Zimmermann A. Transitional liver cell tumors (TLCT) in older children and adolescents: a novel group of aggressive hepatic tumors expressing beta-catenin. Med Pediatr Oncol. 2002;39(5):510-518.
Journal of Hepatocellular Carcinoma

\section{Publish your work in this journal}

The Journal of Hepatocellular Carcinoma is an international, peerreviewed, open access journal that offers a platform for the dissemination and study of clinical, translational and basic research findings in this rapidly developing field. Development in areas including, but not limited to, epidemiology, vaccination, hepatitis therapy, pathology and
Dovepress

molecular tumor classification and prognostication are all considered for publication. The manuscript management system is completely online and includes a very quick and fair peer-review system, which is all easy to use. Visit http://www.dovepress.com/testimonials.php to read real quotes from published authors. 